\title{
Observation of correlated optical and gamma emissions from GRB 081126
}

\author{
A. Klotz \\ Observatoire de Haute-Provence, F-04870 Saint Michel l'Observatoire, France \\ CESR, Observatoire Midi-Pyrénées, CNRS, Université de Toulouse, BP 4346, F-31028 Toulouse Cedex 04, France \\ alain.klotz@cesr.fr \\ B. Gendre \\ LAM/Université de Provence/CNRS, 38 rue Joliot Curie, F-13388 Marseille Cedex 13, France \\ J.L. Atteia \\ LATT, Observatoire Midi-Pyrénées, CNRS, Université de Toulouse, 14 Avenue E. Belin, F-31400 Toulouse, France \\ M. Boër \\ Observatoire de Haute-Provence, F-04870 Saint Michel l'Observatoire, France \\ and \\ D.M. Coward \& A.C. Imerito \\ School of Physics, University of Western Australia, M013, Crawley WA 6009, Australia
}

Received November 1, 2018 /Accepted

\begin{abstract}
We present an analysis of time-resolved optical emissions observed from the gamma-ray burst GRB 081126 during the prompt phase. The analysis employed time resolved photometry using optical data obtained by the TAROT telescope, using BAT data from the Swift spacecraft and time resolved spectroscopy at high energies from the GBM instrument onboard the Fermi spacecraft. The optical emission of GRB 081126 is found to be compatible with the second gamma emission pulse shifted by a positive time-lag of $8.4 \pm 3.9 \mathrm{sec}$. This is the first well resolved observation of a time lag between optical and gamma emissions during a gamma-ray burst. Our observations could potentially provide new constraints on the fireball model for gamma ray burst early emissions. Furthermore, observations of time-lags between optical and gamma ray photons provides an exciting opportunity to constrain quantum gravity theories.
\end{abstract}

Subject headings: gamma-ray : bursts

\section{Introduction}

Because of their extreme luminosity in $\gamma$-rays, gamma ray bursts (GRBs) are a unique probe to high energy regimes where exotic physics is likely to manifest. A fraction of GRBs have been associated with the collapse of massive stars via the association of super- nova signatures observed with the fading GRB optical afterglow e.g. (Hjorth et al. 2003; Stanek et al. 2003). The afterglow most likely originates from an external shock produced as the blast wave from the progenitor collides with the interstellar medium,causing it to slow down and lose energy. Fast moving telescopes linked to GCN notices (Barthelmy 1998) are 
able to record the optical counterpart at the time when the prompt $\gamma$-ray emission is still active. The first positive detection of such event was GRB 990123 (Akerlof et al. 1999). Some other successful detections have been achieved so far (e.g. Rascusin et al. 2008). Two general results have been seen: Either a bright optical emission, uncorrelated to the gammaray light curve, occurred (for 5 to $20 \%$ of GRBs according to Klotz et al. 2009), or that a faint optical emission is correlated with the gamma-ray flares (GRB 050820A, Vestrand et al. 2006). In the former case, these bright optical flashes are often interpreted as the reverse shock signature (Jin \& Fan 2007).

Time lags between $\mathrm{X}$-ray and gamma-ray data are often observed (e.g. Norris et al. 2000). However, this is rare between optical and $\gamma$-rays. As an example, Tang \& Zhang (2006) estimated the most probable time lags for the light curves of GRB 990123 $(5-7 \mathrm{sec})$ and GRB 041219A $(1-5 \mathrm{sec})$. However, the optical data have poor time sampling, putting doubts on these results. Moreover, no lag was noticed for GRB 041219 by Zheng et al. (2006). No lag was reported for GRB 050820A at a level of few seconds Vestrand et al. 2006).

In this letter, we present the measurements of the optical emission observed by TAROT (Klotz et al. 2008) during the prompt $\gamma$-ray activity of GRB 081126 . We show evidence for a positive time lag between optical and $\gamma$-ray light curves.

\section{GRB 081126}

GRB 081126 (Swift BAT trigger 335647, with $\mathrm{T}_{0}=26$ th Nov. 2008, 21:34:10 UT) light curve shows a small precursor starting at $\sim \mathrm{T}_{0}-30 \mathrm{~s}$, peaking at $\sim \mathrm{T}_{0}-18 \mathrm{~s}$, and returning almost to zero at $\mathrm{T}_{0}-7 \mathrm{~s}$ (Sato et al. 2008). The burst features two peaks, the first one at $\sim \mathrm{T}_{0}+1.5 \mathrm{~s}$, reaching its maximum at $\sim \mathrm{T}_{0}+7 \mathrm{sec}$. The second one peaks at $\sim \mathrm{T}_{0}+31.5$ sec. The duration of that burst is $\mathrm{T}_{90}=54 \pm 4 \mathrm{~s}(15-350$ $\mathrm{keV})$. This event was also detected by Konus-Wind (Golenetskii et al. 2008) and the Fermi GBM (Bhat 2008).

The time-averaged spectrum of the first pulse from $\mathrm{T}_{0}$ to $\mathrm{T}_{0}+11 \mathrm{~s}$ is well fit by a Band function with $\mathrm{E}_{\text {peak }}$ $=192 \pm 74 \mathrm{keV}$, alpha $=-0.3 \pm 0.4$, and beta $=-1.6 \pm$ 0.1 . The second pulse from about $\mathrm{T}_{0}+20 \mathrm{~s}$ to $\mathrm{T}_{0}+40$ $\mathrm{s}$ is also well fit by a Band function with $\mathrm{E}_{\text {peak }}=162$ $\pm 77 \mathrm{keV}$, alpha $=-0.3 \pm 0.5$, and beta $=-1.6 \pm 0.1$. The fluence $(8-1000 \mathrm{keV})$ in the two pulses are $(2.7$ $\pm 0.8) \times 10^{-7} \mathrm{erg} \mathrm{cm}^{-2}$ and $(1.9 \pm 0.8) \times 10^{-7} \mathrm{erg} \mathrm{cm}^{-2}$ respectively.

XRT observation reported by Margutti et al. (2008) started 65.7 seconds after the BAT trigger, too late to gather X-ray information of the second peak. The XRT detected a characteristic afterglow emission of the burst. This afterglow was not detected by a quick visual inspection of images taken by TAROT, started $20.6 \mathrm{~s}$ after the burst (Gendre et al. 2008). However, Skvarc \& Mikuz (2008) reported the optical light curve of the afterglow in $\mathrm{R}$ band using the $60 \mathrm{~cm}$ of the Crni Vrh Observatory. Their observations start at $\mathrm{T}_{0}+82 \mathrm{~s}$. They observe a slow rise in optical emission that peaks 200s after the trigger and then fades. This optical afterglow was also reported by Andreev et al. (2008), using the Z-600 telescope of Mt. Terskol observatory, 33 minutes after the burst, and by UVOT (Holland et al. 2008) at $21^{h} 34^{m} 03.59^{s}+48^{\circ} 42^{\prime} 38.3^{\prime \prime}$ (J2000.0). They report that the detection in the U filter, combined with the lack of detections in the UV filters, is consistent with the afterglow having a redshift of approximately $2.4<z<3.8$ (Holland et al. 2008). Unfortunately, no other photometric observations were performed to improve this estimation. From Konus-Wind data, we deduced a pseudo-redshift of $5.3 \pm 1.8$ using the method described in Atteia (2003). The Galactic latitude of the afterglow position is $-2.29^{\circ}$ and the corresponding extinction is $\mathrm{E}(\mathrm{B}-\mathrm{V})=0.782$ mag. according to Schlegel et al. (1998). Assuming $R=3.1$, this gives $\mathrm{A}_{V}=2.6$ and $\mathrm{A}_{R}=2.1 \mathrm{mag}$.

\section{TAROT data}

The first TAROT images were obtained at $\mathrm{T}_{0}+20.1 \mathrm{~s}$ (duration $60 \mathrm{sec}$ ) with the tracking speed adapted to obtain a small trail of a few pixel length. This technique is used in order to obtain temporal informations during the exposure (e.g. Klotz et al. 2006). The spatial sampling is 3.29 arcsec/pix and the FWHM of stars (in the perpendicular direction of the trail) is 2.05 pixels. On the trailed image (see Figure11), the flux of the afterglow is affected by the proximity of NOMAD1 1387-0420537 $(\mathrm{R}=18.1)$ but also by the end of the trail of NOMAD1 1387-0420579 ( $\mathrm{R}=15.48$ hereafter A). This last star lies at 21 arcsec East and 7 arcsec South from the GRB position. As a matter of consequence, the trail of star A (which spreads over 30 arcsec) covers partially the beginning of the trail of the GRB (Fig. 1 top). 
Knowing the position of the afterglow, we first subtracted the trail of the star A within the image. The star NOMAD1 1387-0420302 ( $\mathrm{R}=13.17$, hereafter $\mathrm{B})$ is far enough to other neighbor stars to be used as a trail template to model the star A. We then subtracted this model from the image (using a correct scaling factor to take into account the difference of flux between the stars A and B). The result of the subtraction shows clearly the presence of a dim optical emission (Fig. 1 bottom).

Successive images are $30 \mathrm{~s}$ long exposures tracked on the diurnal motion. Gendre et al. (2008) published only upper limits using TAROT data because it was impossible to detect the optical counterpart so close to the star NOMAD1 1387-0420537 without careful subtraction. The images taken later by TAROT were employed to perform this subtraction. The technique successfully revealed the optical afterglow. In Fig. 2 we display the initial part of the TAROT light curve. We add data from Skvarc \& Mikuz (2008) showing that we can distinguish the early emission that occurred during the gamma activity and the afterglow that followed. A discussion of the afterglow emission process is beyond the scope of this paper and will be presented in Corsi et al. (2009, in preparation).

\section{Data analysis}

From the trailed image, a horizontal profile corresponding to the predicted position of the afterglow gives directly the light curve. We measured the temporal sampling of $6.5 \mathrm{sec} /$ pixel using trails of bright stars. The light curve of the afterglow in the trail presents a flare within 3 pixels (Fig. 3), with a probability of $\sim 10^{-8}$ to be spurious. The probability to observe a cosmic ray at that position is $3.6 \times 10^{-6}$ (estimated from dark fields of the same night). We thus conclude that this flare is real and produced by the burst itself.

Such light curve profiles are affected by the Point spread function (PSF) of TAROT. In order to compare the optical and $\gamma$-ray light curves, we need to convolve the BAT signal by the TAROT PSF. The PSF can be extracted as a vertical profile of a bright non saturated star (seen as the doted curve in the Figure 3). We performed a symetrisation of the PSF shape to be compatible with the hypothesis that the PSF shows no direction effect. Once convolved with the TAROT PSF, the BAT signal corresponding to one peak is very sim- ilar to that of TAROT.

We note at the start of the trail a bright single pixel that could be associated with the end of an optical flare. However, this event is not significant enough to be used in our analysis. Nonetheless, it could be an optical flare related to the first $\gamma$-ray pulse. In the following analysis, we will consider this as a possibility, and thus that the optical flare is correlated to the second $\gamma$-ray pulse, without discarding the possibility that the optical flare is linked to the first $\gamma$-ray pulse.

A $\chi_{v}^{2}$ fit between the optical flare and $\gamma$-ray pulse implies a temporal lag of $+8.4 \pm 3.9 \mathrm{~s}$ (see Fig. 4) at the $97 \%$ confidence $(+38.4 \pm 3.9 \mathrm{~s}$ if the optical flare is related to the first $\gamma$-ray pulse). This is strong evidence for a positive time-lag between the optical and high energy feature. We point out that the exposure time of TAROT images has a better accuracy than $0.1 \mathrm{~s}$ because we use a GPS card triggered by the opening of the shutter, and is not dependent on the computer internal clock variations (Laas-Bourez et al. 2008).

As the TAROT PSF is larger than the BAT second pulse, we also studied the influence of the duration of the BAT pulse modelized by a Gaussian shape, letting free the width of the Gaussian within the fit. The best match of the modelized BAT pulse is a Gaussian spread by sigma $=4.0 \mathrm{sec}$. The $\chi_{v}^{2}$ fit gives the same lag as for the actual BAT pulse meaning that the profile shape of the pulse does not constrain the lag value. The fit remains compatible for Gaussians with sigma lower than $9 \mathrm{sec}$. This means the optical pulse is compatible with a high energy pulse which could have a duration between 0 to $9 \mathrm{sec}$.

The flux of the optical peak observed by TAROT is $0.45 \mathrm{mJy}$. To be compared with the Fermi observations, this value must be corrected for two effects: i) the spread of the flux due to the PSF profile, and ii) the large optical extinction in the $\mathrm{R}$ band. Correcting for all these effects, the optical flux is $\sim 6 \mathrm{mJy}$ at the peak. We used the Band model parameters obtained by the Fermi-GBM (Bhat 2008) to compute the optical flux expected from the high energy band. We derived an expected optical flux of $2.6 \times 10^{-10} \mathrm{Jy}$, which is $\sim 10^{-7}$ times the one observed. Taking account for the uncertainties in the Bhat's alpha parameter the extrapolated flux is always $\sim 10^{-5}$ times the one observed.

\section{Discussion and conclusions}

The analysis of optical and gamma-ray light curves of GRB 081126 reveals: i) the width of the optical 
peak is the same as the gamma-ray peaks, ii) the profile of the optical peak is consistent to the gammaray peaks after correcting for the different PSF, iii) the optical peak occurred $8.4 \pm 3.9$ s (or $+38.4 \pm 3.9$ s) later than the gamma peak. This is the first timelag measured between optical and gamma light curves of a GRB. iv) the gamma-ray flux measured by GBM Fermi, extrapolated to optical energies is $\sim 10^{-7}$ times smaller than the optical flux.

These three results provide potentially new constraints on the theory of prompt GRB emissions. For example, time lags between different energy photons are predicted by quantum gravity in the framework of string theory (e.g. Amelino-Camelia et al. 1998). However, in such a case optical photons should arrive before gamma ones. As we observe the opposite, one can rule out this hypothesis for the GRB 081126's optical lag. Gamma-ray photons comptonization on cold electrons could explain the profile of the optical flare. However, this cannot explain the positive lag observed.

Within the internal shock framework, this temporal lag implies that optical photons were emitted after the $\gamma$-ray ones. However, it is surprising that the flux increases so dramatically during this process. This is not well understood in the standard model for the inelastic internal shock and our results provide new tools for refining the standard model.

B. Gendre acknowledges support from Centre National d'Etudes Spatiales (CNES). The TAROT telescope has been funded by the Centre National de la Recherche Scientifique (CNRS), Institut National des Sciences de l'Univers (INSU) and the Carlsberg Fundation. It has been built with the support of the Division Technique of INSU. We thank the technical staff contributing to the TAROT project, G. Buchholtz, J. Eysseric, M. Merzougui, C. Pollas, P. Richaud and Y. Richaud.

\section{REFERENCES}

Amelino-Camelia, G., Ellis, J., Mavromatos, N.E., et al., 1998, Nature, 393, 763

Andreev M., Sergeev A., Pozanenko, A., 2008, GCNC 8558

Akerlof, C., et al., 1999, Nature, 398, 400

Atteia, J.L., 2003, A\&A 407, L1
Barthelmy, S., in AIP Conf. Proc. 428, GammaRay bursts, ed. C. Meegan \& R. Preece (Berlin: Springer), 99

Bhat, P.N., van der Horst, A.J., 2008, GCNC 8589

Gendre, B., Klotz, A., Atteia, J.L., et al., 2008, GCNC 8555

Golenetskii, S., Aptekar, R., Mazets, E., et al., 2008, GCNC 8562

Hjorth J., et al., 2003, Nature, 423, 847

Holland, S.T., Evans, P.A., Marshall, F.E., et al., 2008, GCNC 8564

Jin, Z.P. \& Fan, Y.Z., 2007, MNRAS 378, 1043

Klotz, A., Gendre, B., Stratta, G., et al., 2006, A\&A, 451, L39

Klotz, A., Boër, M., Eysseric, J., et al., 2008, PASP, 120,1298

Klotz, A., Boër, M., Atteia, J.L., et al., 2009, AJ, in press

Laas-Bourez, M., Boër, M., Blanchet, G., et al. 2008. Cospar 37, 1672

Margutti, R., Beardmore, A.P., Brown, P.J., 2008, CGNC 8554

Norris, J.P., Marani, G.F., Bonnell, J.T., 2000, ApJ 534,248

Rascusin, J.L., Karpov, S.V., Sokolowski, M., et al., 2008, Nature, 455, 183

Sato, G., Barthelmy, S.D., Baumgartner, W.H. , 2008, GCNC 8557

Skvarc, J., Mikuz, H., 2008, GCNC 8569

Schlegel, D.J., Finkbeiner, D.P., Davis, M., 1998, ApJ, 500,525

Stanek K.Z, Matheson, T., Garnavich, P.M., et al. 2003, ApJ, 591, L17

Tang, S.M., Zhang, S.N., 2006, A\&A 456, 141

Vestrand W.T, Wozniak, P.R., Wren, J.A., et al., 2006, Nature 442, 172

Zheng Z., Ye L., Yong-Heng Z., 2006, ApJ 646, L25

This 2-column preprint was prepared with the AAS $\mathrm{L}^{\mathrm{A}} \mathrm{T}_{\mathrm{E}} \mathrm{X}$ macros v5.2. 

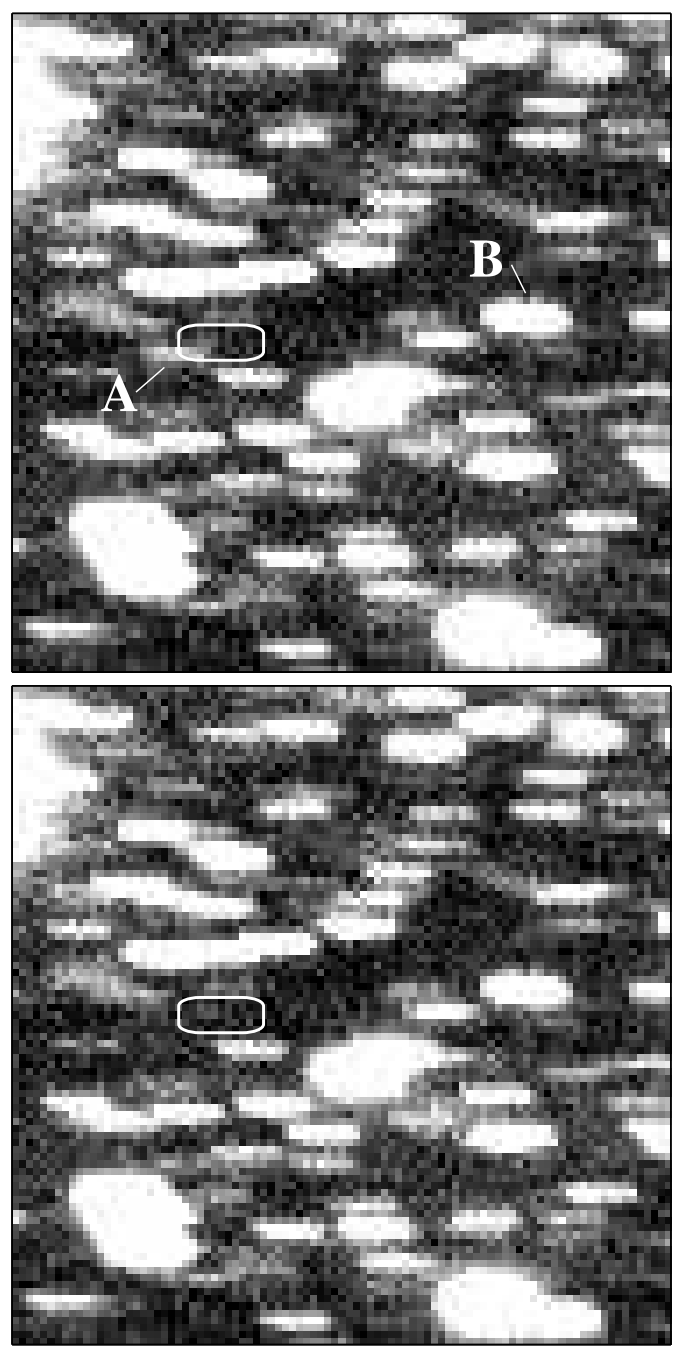

Fig. 1.- Field of GRB 081126. Top: TAROT image taken between 21s and 81s after the GRB trigger. The hour angle velocity was adapted to obtain stars as trails of $\sim 9.2$ pixel length during the 60 s exposure. The theoretical position of the GRB trail is indicated by the white box. The star A covers partly the GRB trail. (see text). Bottom: After subtraction of star A using the model of star B, the trace of the optical emission of the GRB appears in the box The image size is 5 arcmin, North is up, East left.

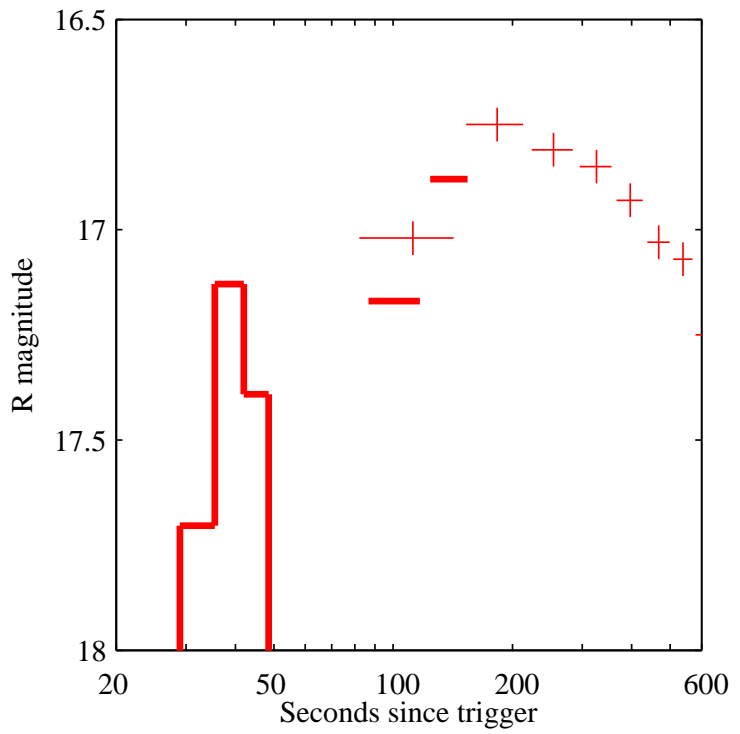

Fig. 2.- Optical light curve of GRB 081126. TAROT optical data are thick bars and observations from Skvarc \& Mikuz (2008) are thin bars (2 sigma level). There are data in the ranges 20-29 and 49$89 \mathrm{sec}$, but with no detection at the limit of $\mathrm{R}=18.0$.

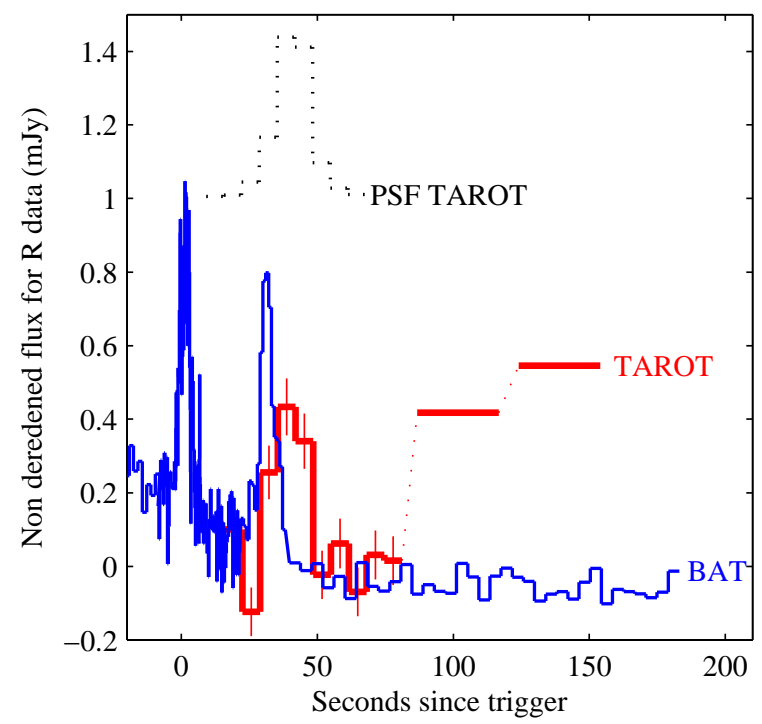

Fig. 3.- Light curves of GRB 081126 measured by BAT and TAROT. The dotted line labeled 'PSFTAROT' stands for the spread of a star equivalent to an instantaneous flash of 0 s duration. This figure appears in colors in the electronic version. 


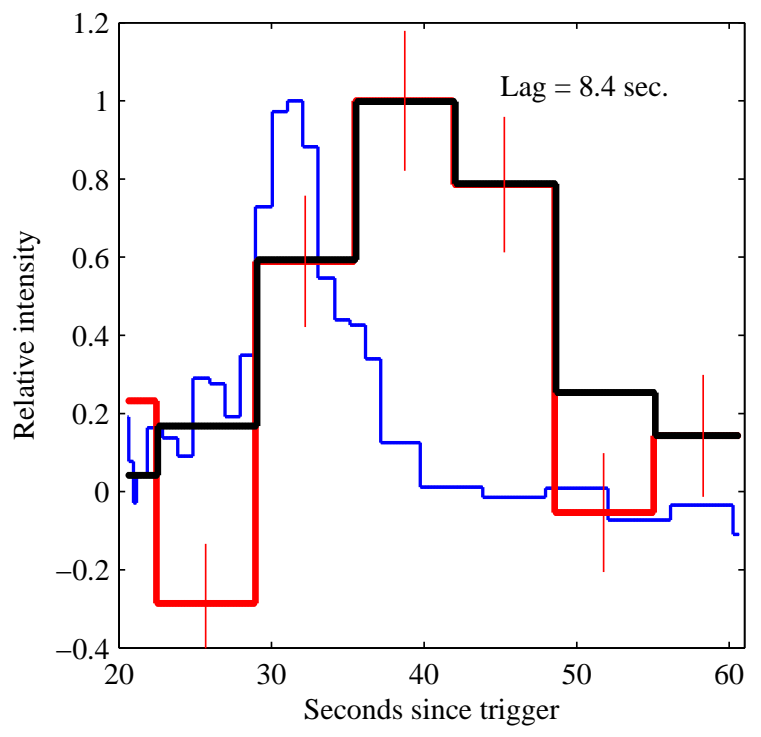

Fig. 4.- The convolution of the peak of BAT light curve (in blue) by the PSF-TAROT shifted by $8.4 \mathrm{~s}$ (in black) compared to the TAROT data (in red). This figure appears in colors in the electronic version. 